\title{
Searching for X-ray luminous "normal” galaxies in 2dFGRS
}

\author{
P. Tzanavaris ${ }^{1}$, I. Georgantopoulos ${ }^{1}$, and A. Georgakakis ${ }^{2}$ \\ 1 Institute of Astronomy \& Astrophysics, National Observatory of Athens, I. Metaxa \& V. Pavlou, Penteli 15236, Greece \\ e-mail: pana@astro.noa.gr \\ 2 Astrophysics Group, Imperial College London, Blackett Laboratory, Prince Consort Rd., London, SW7 2AW, UK
}

Received 2 December 2005 / Accepted 26 February 2006

\section{ABSTRACT}

\begin{abstract}
Aims. We cross-correlated the Chandra XASSIST and XMM-Newton Serendipitous Source Catalogues with the 2 degree Field Galaxy Redshift Survey (2dFGRS) database. Our aim was to identify the most X-ray luminous $\left(L_{\mathrm{X}}>10^{42} \mathrm{erg} \mathrm{s}^{-1}\right)$ examples of galaxies in the local Universe whose X-ray emission is dominated by stellar processes rather than AGN activity ("normal" galaxies), as well as to test the empirical criterion $\log \left(f_{\mathrm{X}} / f_{\mathrm{O}}\right)<-2$ for separating AGN from NGs. We also performed a similar search in two nearby-galaxy samples from the literature.

Methods. With XMM-Newton (Chandra) we covered an area of $\sim 8.2(\sim 5.8) \mathrm{deg}^{2}$ down to a flux limit of $\sim 10^{-15}(\sim 1.6 \times$ $10^{-15}$ ) erg cm $\mathrm{cm}^{-2} \mathrm{~s}^{-1}$. We classified $2 d F G R S$ spectra using emission-line intensity ratios.

Results. We found 18 (20) 2dFGRS galaxies with XMM-Newton (Chandra), which constitute our XMM-Newton-2dFGRS (Chandra$2 d F G R S$ ) correlation sample. We classified $62 d F G R S$ spectra as star-forming, H II nuclei, and 2 spectra as possible H II nuclei. The rest of the objects are absorption-line galaxies and AGN, including 3 possible LINERs. No luminous "normal" galaxies have been found, but out of 19 "normal" galaxies in this sample, $5 \mathrm{H}$ II and 3 absorption-line galaxies have $\log \left(f_{\mathrm{X}} / f_{\mathrm{O}}\right)>-2$. Furthermore, all 44 galaxies in the first literature sample have $\log \left(f_{\mathrm{X}} / f_{\mathrm{O}}\right)<-2$ and $L_{\mathrm{X}}<10^{42} \mathrm{erg} \mathrm{s}^{-1}$. In the second literature sample, out of a total of 170 "normal" galaxies, we found 16 galaxies with $\log \left(f_{\mathrm{X}} / f_{\mathrm{O}}\right)>-2$, the majority of which are massive ellipticals. Three of these have $L_{\mathrm{X}}>10^{42} \mathrm{erg} \mathrm{s}^{-1}$.

Conclusions. We found no luminous "normal" galaxies in our XMM-Newton-2dFGRS and Chandra-2dFGRS correlation samples. We found three such galaxies in the second literature sample. Considering all samples, we find that $\log \left(f_{\mathrm{X}} / f_{\mathrm{O}}\right)<-2$ criterion seems to select primarily against the brightest, massive ellipticals.
\end{abstract}

Key words. X-rays: galaxies - galaxies: starburst - galaxies: active

\section{Introduction}

Galaxies hosting an active galactic nucleus (AGN) have long been known to be sources of copious amounts of X-ray emission. On the other hand, galaxies which are "normal" (NGs), in the sense that they are not AGN-dominated, have also been studied in detail. The source of X-ray emission in these systems is diffuse hot gas and/or X-ray binary stars. In the most massive early-type galaxies the X-ray emission is dominated by the hot interstellar medium with temperatures $k T \sim 1 \mathrm{keV}$. A smaller fraction of the observed X-ray luminosity is due to low mass $\mathrm{X}$-ray binaries associated with the older stellar population. In late-type galaxies, the $\mathrm{X}$-ray emission originates in hot gas with temperature $k T \sim 1 \mathrm{keV}$, which is heated by supernova remnants, as well as in a mixture of low- and high-mass X-ray binaries (see Fabbiano 1989, for a review). The diffuse hot gas contributes significantly in the soft X-ray band $(<2 \mathrm{keV})$, while the X-ray binary systems are responsible for the bulk of the emission at harder energies (e.g., Stevens et al. 2003). The integrated $\mathrm{X}$-ray emission of "normal" galaxies is believed to be a good indicator of the star-formation activity in these systems (e.g., Gilfanov et al. 2004), at least if the star-formation rate is not too low.

The X-ray luminosity of NGs is usually weak, $\lesssim 10^{42} \mathrm{erg} \mathrm{s}^{-1}$, i.e., a few orders of magnitude below that of powerful AGN (Moran et al. 1999; Zezas et al. 1998). As a result, observed $\mathrm{X}$-ray fluxes are faint and, until recently, only the very local systems $(<100 \mathrm{Mpc})$ were accessible to X-ray missions. With the new generation of X-ray missions, Chandra and XMM-Newton, the situation has changed dramatically. The Chandra Deep Fields North and South (CDF-N, CDF-S; Alexander et al. 2003; Giacconi et al. 2002) have reached fluxes $f(0.5-2.0 \mathrm{keV}) \sim$ $10^{-17} \mathrm{erg} \mathrm{cm}^{-2} \mathrm{~s}^{-1}$, thus providing the first ever X-ray-selected sample of NGs at cosmologically interesting redshifts. Using the $2 \mathrm{Ms}$ CDF-North, Hornschemeier et al. (2003) provided a sample of $43 \mathrm{NG}$ candidates for which optical spectroscopic observations are available. These galaxies have X-rayto-optical flux ratios $\log \left(f_{\mathrm{X}} / f_{\mathrm{O}}\right) \lessgtr-2$, which these authors use as an empirical boundary, separating quiescent NGs from AGN. Norman et al. (2004) extended this study and identified over 100 NG candidates in the combined CDF-N and CDF-S, although optical spectroscopic data are available only for a fraction of these objects. However, these authors have included NGs with $\log \left(f_{\mathrm{X}} / f_{\mathrm{O}}\right)>-2$. On the other hand, Georgakakis et al. (2003) and Georgakakis et al. (2004a) have identified NGs with $\log \left(f_{\mathrm{X}} / f_{\mathrm{O}}\right) \approx-2$. Within the framework of the "Needles in the Haystack Survey" (NHS), Georgakakis et al. (2004b) and Georgantopoulos et al. (2005) combined XMM-Newton data with the Sloan Digital Sky Survey and used several selection criteria, including $\log \left(f_{\mathrm{X}} / f_{\mathrm{O}}\right)<-2$, to identify 28 NG candidates. By combining this sample with $18 z<0.2$ galaxies from the CDFs, these authors constructed the first local X-ray luminosity function of NGs. However, it must be stressed that this result depends on the completeness of NG samples, which, in turn, may be biasing to quiescent systems and selecting against $\mathrm{X}$-ray luminous (with X-ray luminosities $L_{\mathrm{X}}>10^{42} \mathrm{erg} \mathrm{s}^{-1}$ ) 
starbursts and massive ellipticals, which are likely to show $-2<\log \left(f_{\mathrm{X}} / f_{\mathrm{O}}\right)<-1$ (Alexander et al. 2003). Indeed, the local luminosity function of Georgantopoulos et al. (2005) agrees well with that of Norman et al. (2004) at the faint end, but disagrees at the bright end. If bright galaxies are missed due to the $\log \left(f_{\mathrm{X}} / f_{\mathrm{O}}\right)<-2$ criterion, this might explain the discrepancy. Alternatively, the Norman et al. (2004) may suffer from AGN contamination.

It is thus imperative to understand the significance of this bias and to resolve the controversy to constrain the local luminosity function, which also provides a local "anchor point" for investigating luminosity function evolution.

In the work described in this paper, we searched for luminous NGs by performing a cross-correlation between, on the one hand, two large X-ray catalogues, and, on the other hand, the 2 degree field galaxy redshift survey $(2 d F G R S)$. Our aim was twofold:

1. to search for X-ray luminous $\left(L_{\mathrm{X}}>10^{42} \mathrm{erg} \mathrm{s}^{-1}\right)$, especially star-forming, galaxies, and

2. to test the empirical criterion $\log \left(f_{\mathrm{X}} / f_{\mathrm{O}}\right)<-2$ for separating NGs from AGN so as to address the discrepancy described above.

Throughout this paper we use a Hubble parameter $H_{0}=$ $72 \mathrm{~km} \mathrm{~s}^{-1} \mathrm{Mpc}^{-1}$, matter density $\Omega_{\mathrm{M}}=0.3$, and a cosmological constant $\Omega_{\Lambda}=0.7$.

\section{Data}

\section{1. $2 d f G R S$ data}

The $2 d F G R S$ is a joint UK-Australian project, which obtained spectra for 245591 objects brighter than a nominal extinctioncorrected magnitude limit of $b_{\mathrm{J}}=19.45$, over an area of $\approx 1500 \mathrm{deg}^{2}$. This survey is fairly uniform, with a known incompleteness at the bright end ( $b_{\mathrm{J}} \lesssim 16$, Norberg et al. 2002). Reliable redshifts were obtained with the aim of providing a detailed three-dimensional picture of galaxy population and large scale structure in the local Universe (Colless et al. 2003, 2001). We used heliocentric-corrected redshift values given in the FITS headers of the $2 d F G R S$ spectra. The galaxies are selected from the extended APM Galaxy Survey in three regions: a strip near the north galactic pole, a strip near the south galactic pole, and random fields around this.

For our purposes, the $2 d F G R S$ presents the advantage that its depth allows detection of galaxies up to $\log \left(f_{\mathrm{X}} / f_{\mathrm{O}}\right) \lesssim-1$ for $f_{\mathrm{X}} \approx$ few $\times 10^{-14}$ (see Fig. 2).

\subsection{XMM-Newton observations}

We used XMM-Newton archival observations from the XMMNewton Serendipitous Source Catalogue, Version 1.1.0, (1XMM), whose fields overlap those from the final data release of the $2 d F G R S$. The catalogue contains more than 50000 single sources in a total of 585 fields. Specifically, we have cross-correlated the celestial coordinates (right ascension and declination, J2000) of sources in the two data sets to detect optical and X-ray counterparts. The cross-correlation was performed independently for each of the three CCDs of the EPIC camera because in some cases a source may not have been recorded in all three CCDs. The X-ray band was restricted to $0.5-2.0 \mathrm{keV}$ as galaxies are preferentially soft X-ray emitters (e.g., Levenson et al. 2001). We set the detection likelihood parameter to $\gtrsim 7$ and the matching radius to $6^{\prime \prime}$.
This corresponds to $\sim 3 \sigma$ in terms of on-axis XMM/EPIC positional uncertainty. Off-axis sources suffer from vignetting, and such sources will have increased positional uncertainty. This is further increased for sources whose X-ray emission is dominated by an off-nuclear component.

We obtained 18 sources which have been detected both by $2 d F G R S$ and by XMM/EPIC. The sources are detected in $42 X M M$-Newton fields. The area covered for this sample is $\sim 8.2 \mathrm{deg}^{2}$ to a flux limit of $\sim 10^{-15} \mathrm{erg} \mathrm{cm}^{-2} \mathrm{~s}^{-1}$. The largest separation between an X-ray and an optical position in this sample is 5.1" . All X-ray/optical counterparts were checked visually. For all sources, we estimate the probability of detecting an optical counterpart by chance to be less than $1 \%$ for all sources. These $18 \mathrm{X}$-ray/optical pairs form our XMM-Newton-2dFGRS correlation sample.

We calculated X-ray fluxes, $f_{\mathrm{X}}$, after taking into account the column density of Galactic neutral hydrogen, $N_{\mathrm{H}}$, along the line of sight to each observed source. Further, we calculated the $\mathrm{X}$-ray luminosity, $L_{\mathrm{X}}$, by using source redshifts, $z$, and X-ray fluxes, assuming power law spectra with a photon index $\Gamma=1.8$.

We obtained hardness ratio values, HR, from the 1XMM catalogue, using unvignetted count rates in the energy bands $0.5-2.0 \mathrm{keV}(\mathrm{S})$ and $2.0-4.5 \mathrm{keV}(\mathrm{H})$, so that

$\mathrm{HR} \equiv \frac{\mathrm{H}-\mathrm{S}}{\mathrm{H}+\mathrm{S}}$

Details of our XMM-Newton-2dFGRS correlation sample are given in Table 1.

\subsection{Chandra observations}

We used Chandra archival observations from the Chandra XAssist catalogue (version 3). XAssist (http:// www.xassist.org, Ptak \& Griffiths 2003) is a NASA AISR-funded project (NAG 5-809) for the automation of X-ray astrophysics, with emphasis on galaxies. The XAssist catalogue comprises more than 40000 single X-ray sources detected in the 0.3-8.0 keV band in a total of 913 fields. We cross-correlated celestial coordinates in the XAssist and 2dFGRS catalogues for overlapping fields from the two databases by using a procedure similar to that used to obtain the XMM-Newton-2dFGRS correlation sample. We used a matching radius of $3^{\prime \prime}$, which corresponds to $\gtrsim 3 \sigma$ in terms of on-axis positional uncertainty for ACIS. As in the case of XMM-Newton, we also need to account for a large increase in this uncertainty for off-axis and off-nuclear sources.

We obtained 20 sources for which the optical and X-ray sources are separated by less than $3^{\prime \prime}$, the largest X-ray/optical offset being 2.9". The sources are detected in 58 distinct fields. The area covered for this sample is $\sim 5.8 \mathrm{deg}^{2}$ to a flux limit of $\sim 10^{-15.2} \mathrm{erg} \mathrm{cm}^{-2} \mathrm{~s}^{-1}$. For each source, we calculated $L_{\mathrm{X}}$ by using $z$ and $f_{\mathrm{X}}$. We used the value of $f_{\mathrm{X}}$ provided by XASSIST.

We calculated HRs using the original ACIS event files and the soft (hard) energy band $0.3-2.0 \mathrm{keV}(2.0-8.0 \mathrm{keV})$.

As in the previous section, the sources were also checked visually. The probability of detecting an optical counterpart by chance is, once more, less than $1 \%$.

Details of our Chandra-2dFGRS correlation sample are given in Table 2 .

\section{Classification of sources}

In what follows, we refer to the XMM-Newton and Chandra correlation samples together as "the $2 d F G R S$ correlation 
Table 1. The XMM-Newton/2dFGRS correlation sample. Shown from left to right are sample identification number, $2 d F G R S$ database name, right ascension and declination for the X-ray source, offset between X-ray and optical source, $b_{\mathrm{J}}$ magnitude from $2 d F G R S$, X-ray flux, redshift from $2 d F G R S$, logarithm of X-ray luminosity, logarithmic X-ray-to-optical flux ratio, hardness ratio and error, and, in the last column, suggested galaxy type. For this column, the following abbreviations hold: A: absorption line galaxy; F: featureless $2 d F G R S$ spectrum; G: source appears in group; H II: H II nucleus. A question mark indicates that a classification is not possible. Question marks after a classification indicate a high degree of uncertainty.

\begin{tabular}{|c|c|c|c|c|c|c|c|c|c|c|c|c|}
\hline ID & Name & $\begin{array}{c}\alpha_{\mathrm{X}} \\
(\mathrm{J} 2000)\end{array}$ & $\begin{array}{c}\delta_{\mathrm{X}} \\
(\mathrm{J} 2000)\end{array}$ & $\begin{array}{c}\delta_{\mathrm{XO}} \\
\left({ }^{\prime \prime}\right)\end{array}$ & $b_{\mathrm{J}}$ & $\begin{array}{c}f_{\mathrm{X}} / 10^{-14} \\
(\mathrm{cgs})\end{array}$ & $z$ & $\begin{array}{c}\log L_{X} \\
(\operatorname{cgs})\end{array}$ & $\log \left(f_{\mathrm{X}} / f_{\mathrm{O}}\right)$ & HR & \pm & Type \\
\hline 1 & TGS548Z244 & 235627.68 & -343535.8 & 0.65 & 15.52 & 1.700 & 0.0479 & 40.94 & -2.09 & -1.00 & 0.11 & $\mathrm{~A}$ \\
\hline 2 & TGS549Z357 & 235610.72 & -344942.1 & 3.20 & 18.73 & 1.170 & 0.2399 & 42.26 & -0.97 & -0.74 & 0.16 & $\mathrm{~F}, \mathrm{G}$ \\
\hline 3 & TGS617Z146 & 005818.33 & -355548.1 & 3.12 & 18.01 & 0.375 & 0.0479 & 40.28 & -1.75 & -0.57 & 0.15 & H II \\
\hline 4 & TGS210Z018 & 005551.51 & -272609.7 & 3.19 & 19.12 & 4.555 & 0.2125 & 42.74 & -0.22 & -0.50 & 0.24 & $\mathrm{~F}$ \\
\hline 5 & TGS327Z003 & 215106.12 & -30 2427.1 & 1.72 & 18.83 & 2.080 & 0.1373 & 42.00 & -0.68 & -0.77 & 0.17 & $\mathrm{~A}, \mathrm{G}$ \\
\hline 6 & TGS063Z133 & 222151.26 & -244530.7 & 0.98 & 19.08 & 2.470 & 0.0777 & 41.54 & -0.51 & -0.26 & 0.09 & AGN \\
\hline 7 & TGS120Z043 & 223532.80 & -260605.6 & 3.83 & 15.37 & 0.321 & 0.0192 & 39.40 & -2.87 & -0.62 & 0.15 & H II \\
\hline 8 & TGN296Z199 & 104444.83 & -012017.7 & 1.35 & 18.73 & 2.230 & 0.1846 & 42.29 & -0.69 & -0.71 & 0.04 & $?$ \\
\hline 9 & TGN295Z042 & 104419.91 & -012426.7 & 1.97 & 18.69 & 0.158 & 0.0610 & 40.13 & -1.86 & -0.37 & 0.17 & Н II \\
\hline 10 & TGN295Z067 & 104352.59 & -011740.1 & 2.97 & 14.69 & 3.330 & 0.0262 & 40.70 & -2.13 & -0.75 & 0.03 & H II \\
\hline 11 & TGN448Z020 & 115129.69 & +014831.7 & 5.81 & 18.80 & 0.211 & 0.1581 & 41.13 & -1.68 & -0.80 & 0.17 & $\mathrm{~F}$ \\
\hline 12 & TGN388Z113 & 122947.33 & +015403.5 & 1.03 & 19.04 & 1.360 & 0.1577 & 41.94 & -0.78 & -0.77 & 0.11 & $?$ \\
\hline 13 & TGN387Z032 & 122858.43 & +02 1127.2 & 0.14 & 16.64 & 0.500 & 0.0775 & 40.84 & -2.18 & -1.00 & 0.07 & A \\
\hline 14 & TGN387Z056 & 122817.84 & +02 1229.3 & 0.55 & 18.86 & 3.850 & 0.1203 & 42.16 & -0.40 & -0.66 & 0.11 & $?$ \\
\hline 15 & TGN387Z067 & 122807.61 & +020252.1 & 0.66 & 18.48 & 0.694 & 0.0903 & 41.12 & -1.30 & -0.61 & 0.19 & $?$ \\
\hline 16 & TGN266Z089 & 133139.59 & -014826.2 & 4.79 & 18.23 & 0.290 & 0.0749 & 40.57 & -1.77 & -0.26 & 0.20 & Н II \\
\hline 17 & TGS924Z253 & 225228.19 & -174916.8 & 0.78 & 19.24 & 3.290 & 0.1331 & 42.16 & -0.31 & -0.63 & 0.05 & $?$ \\
\hline 18 & TGS924Z246 & 225231.62 & -174632.4 & 0.60 & 18.44 & 0.853 & 0.0689 & 40.97 & -1.22 & -0.43 & 0.23 & H II \\
\hline
\end{tabular}

Table 2. The Chandra/2dFGRS correlation sample. Column details are as in the previous figure.

\begin{tabular}{|c|c|c|c|c|c|c|c|c|c|c|c|c|}
\hline ID & Name & $\begin{array}{c}\alpha_{\mathrm{X}} \\
(\mathrm{J} 2000)\end{array}$ & $\begin{array}{c}\delta_{\mathrm{X}} \\
(\mathrm{J} 2000)\end{array}$ & $\begin{array}{c}\delta_{\mathrm{XO}} \\
\left({ }^{\prime \prime}\right)\end{array}$ & $b_{\mathrm{J}}$ & $\begin{array}{c}f_{\mathrm{X}} / 10^{-14} \\
(\mathrm{cgs})\end{array}$ & $z$ & $\begin{array}{c}\log L_{X} \\
(\mathrm{cgs})\end{array}$ & $\log \left(f_{\mathrm{X}} / f_{\mathrm{O}}\right)$ & HR & \pm & Type \\
\hline 1 & TGS432Z052 & 235827.21 & -324103.3 & 0.23 & 19.20 & 6.36 & 0.2394 & 42.99 & -0.50 & +0.39 & 0.04 & $\mathrm{AGN}^{1}$ \\
\hline 2 & TGS522Z150 & 025714.04 & -331827.5 & 0.82 & 17.93 & 1.84 & 0.1093 & 41.72 & -1.54 & +0.23 & 0.11 & $\mathrm{AGN}^{1}$ \\
\hline 3 & TGS212Z026 & 005824.51 & -272923.2 & 0.78 & 16.83 & 0.36 & 0.0977 & 40.91 & -2.70 & -0.63 & 0.24 & H II? \\
\hline 4 & TGS211Z082 & 005651.78 & -272856.3 & 1.44 & 18.90 & 4.22 & 0.2144 & 42.71 & -0.80 & +0.61 & 0.11 & $\mathrm{AGN}^{1}$ \\
\hline 5 & TGS243Z005 & 033246.89 & -274213.3 & 2.52 & 17.53 & 0.39 & 0.1028 & 41.00 & -2.38 & -0.54 & 0.12 & A \\
\hline 6 & TGS407Z114 & 220136.09 & -315323.2 & 1.63 & 18.06 & 1.63 & 0.0972 & 41.56 & -1.55 & +0.24 & 0.03 & $\mathrm{AGN}^{1}$ \\
\hline 7 & TGS132Z150 & 235139.38 & -260502.5 & 2.90 & 18.00 & 9.17 & 0.2346 & 43.13 & -0.82 & -0.61 & 0.01 & LINER? \\
\hline 8 & TGN163Z121 & 105650.04 & -033342.8 & 1.10 & 17.69 & 0.69 & 0.0488 & 40.56 & -2.07 & -0.56 & 0.07 & A (noisy) \\
\hline 9 & TGN163Z123 & 105648.84 & -033725.8 & 0.73 & 18.54 & 0.41 & 0.1816 & 41.55 & -1.96 & -0.78 & 0.10 & A \\
\hline 10 & TGN071Z177 & 124902.23 & -054933.8 & 1.43 & 18.34 & 0.60 & 0.0485 & 40.50 & -1.87 & -0.32 & 0.08 & H II? \\
\hline 11 & TGN206Z127 & 141249.69 & -030719.7 & 2.59 & 18.09 & 0.94 & 0.0748 & 41.08 & -1.77 & -0.43 & 0.13 & AGN? \\
\hline 12 & TGN440Z057 & 112320.17 & +013809.9 & 1.84 & 18.66 & 0.07 & 0.1259 & 40.47 & -2.66 & -0.68 & 0.25 & A \\
\hline 13 & TGN382Z123 & 120427.15 & +015346.2 & 2.69 & 14.25 & 3.10 & 0.0197 & 40.42 & -2.79 & -0.80 & 0.01 & A \\
\hline 14 & TGN243Z240 & 115545.38 & -014130.3 & 0.70 & 19.00 & 1.79 & 0.2476 & 42.48 & -1.13 & -0.57 & 0.08 & LINER? \\
\hline 15 & TGN247Z076 & 121601.62 & -003733.6 & 0.46 & 17.70 & 0.25 & 0.1214 & 40.98 & -2.50 & -1.00 & 0.24 & A \\
\hline 16 & TGN318Z209 & 121553.29 & -003606.9 & 1.71 & 18.00 & 0.23 & 0.1193 & 40.90 & -2.42 & -0.39 & 0.21 & A \\
\hline 17 & TGN336Z169 & 134452.88 & +000520.4 & 0.81 & 17.38 & 77.8 & 0.0876 & 43.14 & -0.14 & -0.63 & 0.02 & AGN? ${ }^{2}$ \\
\hline 18 & TGN336Z187 & 134428.34 & +000147.2 & 0.71 & 18.12 & 0.56 & 0.1351 & 41.43 & -1.98 & -1.00 & 0.48 & A \\
\hline 19 & TGN275Z203 & 141234.68 & -003500.1 & 0.49 & 18.16 & 30.4 & 0.1269 & 43.09 & -0.24 & -0.74 & 0.07 & $\mathrm{AGN} ?^{3}$ \\
\hline 20 & TGS906Z501 & 232519.74 & -120726.2 & 1.77 & 15.89 & 4.94 & 0.0824 & 41.89 & -1.93 & -0.64 & 0.002 & LINER? \\
\hline
\end{tabular}

1: Obscured AGN suggested by high $N_{\mathrm{H}}$ value.

2: Suggested by an $\mathrm{H} \beta F W H M \sim 2000 \mathrm{~km} \mathrm{~s}^{-1}$.

3: Suggested by an $\mathrm{H} \beta F W H M \sim 1500 \mathrm{~km} \mathrm{~s}^{-1}$.

sample". We now describe our approach for identifying NGs in this sample.

For optical spectra it has been shown that empirical emission-line intensity ratios may be used as a diagnostic of AGN activity (BPT diagrams, Baldwin et al. 1981). We adopted the classification scheme of Ho et al. (1997), which is based on the diagnostic diagrams proposed by Veilleux \& Osterbrock (1987). Ho et al. (1997) used the line ratios [O III] 5007/H $\beta$, [O I] 6300/H $\alpha$, [N II] 6583/H $\alpha$, and [S II] 6716, 6731/H $\alpha$, which are least sensitive to dust reddening and flux calibration because the wavelength separation between members of each line pair is small. Additionally, since the ratios involve a line of only one element and an H I Balmer line, they are less abundancesensitive. We used this as our primary method of classification. Unfortunately, absolute flux calibration for $2 d F G R S$ fibres, which can differ substantially in their throughput, cannot be done reliably. For this reason, we were unable to perform subtraction of stellar templates, which depends crucially on the shape of the galaxy spectrum. As a result, we only classified galaxies as "normal" (or not), when all four of the line-intensity ratios 
Table 3. NGs with $\log \left(f_{\mathrm{X}} / f_{\mathrm{O}}\right)>-2$. Groups of rows separated by horizontal lines correspond to the sample indicated in the first column. $2 d F G R S$ stands for the combined correlation samples 2dFGRS-XMM-Newton and 2dFGRS-Chandra. Each row corresponds to the NG type indicated in the second column. Each of the last three columns gives the fraction of NGs with $\log \left(f_{\mathrm{X}} / f_{\mathrm{O}}\right)>-2$ in the $\log L_{\mathrm{X}}$ region indicated at the top of the column. Note that the second of these columns includes NGs from the $\log L_{\mathrm{X}}<42$ column which also have $\log L_{\mathrm{X}}>41$. Thus, the numerator gives the number of NGs of a given type in a given sample and a given $\log L_{X}$ range, and the denominator the total number of NGs in the same sample and $\log L_{\mathrm{X}}$ range. Galaxies for which only upper limit information is available have not been taken into account. Empty entries indicate that no NGs of this type have been found. Galaxy labels are as in Tables 1 and 2. Additionally, "sb"rq stands for starburst and "E" for elliptical.

\begin{tabular}{llccc}
\hline \hline Sample & NG type & $\log L_{\mathrm{X}}<42$ & $41<\log L_{\mathrm{X}}<42$ & $\log L_{\mathrm{X}} \geq 42$ \\
\hline $2 d F G R S$ & H II & $4 / 18$ & & \\
& H II? & $1 / 18$ & & \\
& $\mathrm{~A}$ & $2 / 18$ & $2 / 2$ & $1 / 1$ \\
\hline Zezas (2001) & any & 0 & 0 & 0 \\
\hline Fabbiano et al. (1992) & sb & $2 / 167$ & $1 / 26$ & \\
& $\mathrm{E}$ & $11 / 167$ & $11 / 26$ & $3 / 3$ \\
\hline
\end{tabular}

unambiguously suggested this. If only some lines were observable, the classification is only tentative, which is denoted by a question mark after the type entry in the sample tables. Finally, some spectra were tentatively classified as LINERS, according to the classification criteria of Ho et al. (1997).

In the case of sources $1,2,4$, and 6 from the Chandra$2 d F G R S$ correlation sample, the above method failed. Using the tool PIMMS ${ }^{1}$, we carried out tests to estimate the hydrogen column density needed to obtain the observed hardness ratio, assuming the source flux and redshift, as well as a power law spectrum with photon index around $\Gamma=1.8$. Our tests showed that for all sources $N_{\mathrm{H}}>10^{22}$, suggesting obscured AGN, as indicated in Table 2. However, better optical observations are needed to clarify the situation further, for the following reasons. For source 1, the spectrum is of poor quality, whilst the $\mathrm{H} \alpha$ line is not covered. For source 2, the $\mathrm{H} \alpha$ and [N II] lines appear suppressed, but it is unclear whether this has a physical origin or is a data reduction artefact. For source 4, there is a hint of a broad $\mathrm{H} \alpha$ line, which only partially falls within the $2 d F G R S$ spectrum. In the case of source 6 , the $\mathrm{H} \alpha$ line appears absorbed and the [N II] line falls outside the spectrum.

The emission-line ratio method also failed for sources 17 and 19. However, as the optical spectra of these sources show very broad $\mathrm{H} \alpha$ or $\mathrm{H} \beta$ emission lines $\left(>1000 \mathrm{~km} \mathrm{~s}^{-1}\right)$, the sources were also classified as AGN, and this is indicated in Table 2.

Further, to separate NGs from AGN, it is often assumed that for NGs, the X-ray-to-optical flux ratio obeys $\log \left(f_{\mathrm{X}} / f_{\mathrm{O}}\right)<$ -2 , i.e., is two orders of magnitude lower than for typical AGN. The X-ray luminosity is similarly assumed to obey $L_{\mathrm{X}} \lesssim$ $10^{42} \mathrm{erg} \mathrm{s}^{-1}$ (Fabbiano 1989).

For the $2 d F G R S$ correlation sample, we estimated $\log \left(f_{\mathrm{X}} / f_{\mathrm{O}}\right)$ from the relation

$\log \frac{f_{\mathrm{X}}}{f_{\mathrm{O}}}=\log f_{\mathrm{X}}(0.3-3.5 \mathrm{keV})+0.4 V+5.37$

(Stocke et al. 1991). The X-ray flux for this relation was calculated from the $0.2-8.0 \mathrm{keV}(0.5-2.0 \mathrm{keV})$ flux of our XASSIST (1XMM) dataset, assuming a power law index $\Gamma=1.8$. The $V$ magnitude was calculated from the $b_{\mathrm{j}}$ magnitude listed in the $2 d F G R S$ database and used to select the objects optically. The conversion was performed using the relations

$b_{\mathrm{j}}=B-0.28(B-V)$,

which holds for the $2 d F G R S$ galaxies and

$B-V=0.53$

${ }^{1}$ http://heasarc.gsfc.nasa.gov/Tools/w3pimms.html
(Fukugita et al. 1995, Table 3a). This is the average $B-V$ value for nearby galaxies with types in the range Sab, Sbc, Scd, and Irr.

We stress that we did not use either $L_{\mathrm{X}}$ or $\log \left(f_{\mathrm{X}} / f_{\mathrm{O}}\right)$ to classify sources. On the contrary, as explained above, where possible, we first classified sources as NGs or AGN using optical criteria. Subsequently, we used $L_{X}$ to look for luminous NGs, and checked whether the classification agreed with the empirical AGN/NG break at $\log \left(f_{\mathrm{X}} / f_{\mathrm{O}}\right) \sim-2$.

\section{Results and discussion}

Results for the $2 d F G R S$ correlation sample are summarised in Tables 1 and 2. We found six NGs, which fall under the category of H II nuclei, and two tentative H II nuclei. Five galaxies are classified as AGN, and three as tentative AGN. There are eleven absorption-line galaxies, presumably elliptical, for which the $L_{\mathrm{X}}, \log \left(f_{\mathrm{X}} / f_{\mathrm{O}}\right)$ and HR values, considered together, suggest that these are also not AGN-dominated. A further three sources are tentatively classified as LINERs. For the rest of the galaxies, there is not enough information to make even a tentative classification. As can be seen in Tables 1 and 2, all "non-A" NGs have $\log L_{X}<42$. In that sense, no luminous star-forming galaxies have been found. However, four of the H II nuclei, one of the tentative $\mathrm{H}$ II nuclei, as well as three absorption-line galaxies have $\log \left(f_{\mathrm{X}} / f_{\mathrm{O}}\right)>-2$ (Table 3$)$, i.e., overall, $\sim 40 \%$ of NGs found appear to have $\log \left(f_{\mathrm{X}} / f_{\mathrm{O}}\right)>-2$.

A plot of $\log \left(f_{\mathrm{X}} / f_{\mathrm{O}}\right)$ versus $L_{\mathrm{X}}$ is shown in Fig. 1. Apart from our XMM-Newton and Chandra results, for comparison and completeness purposes we have plotted data from the following sources:

1. The nearby star-forming galaxy sample compiled by Zezas (2001):

This comprises 44 galaxies detected by ROSAT PSPC, spanning the luminosity range $L_{\mathrm{X}}(0.1-2.4 \mathrm{keV}) \approx 4 \times 10^{37}-3 \times$ $10^{41} \mathrm{erg} \mathrm{s}^{-1}$. Galaxies in this sample have been classified on the basis of high quality nuclear spectra from Ho et al. (1997).

2. The nearby galaxy sample of Fabbiano et al. (1992):

The galaxies have been observed with the Einstein observatory and comprise all morphological types. Galaxies flagged as AGN hosts in the original sample have been excluded. We have also carried out a further literature search to exclude more AGN from the final plotted sample. Galaxies for which only upper limit $f_{\mathrm{X}}$ and $L_{\mathrm{X}}$ information is available are plotted with downward-pointing arrows. We used the $L_{\mathrm{X}}$ values from Fabbiano et al. (1992), after scaling for $H_{0}=72 \mathrm{~km} \mathrm{~s}^{-1} \mathrm{Mpc}^{-1}$. The $\log \left(f_{\mathrm{X}} / f_{\mathrm{O}}\right)$ values plotted were computed as explained in Sect. 3, using the $0.2-4.0 \mathrm{keV}$ flux and $B$ magnitude information from Fabbiano et al. (1992), 


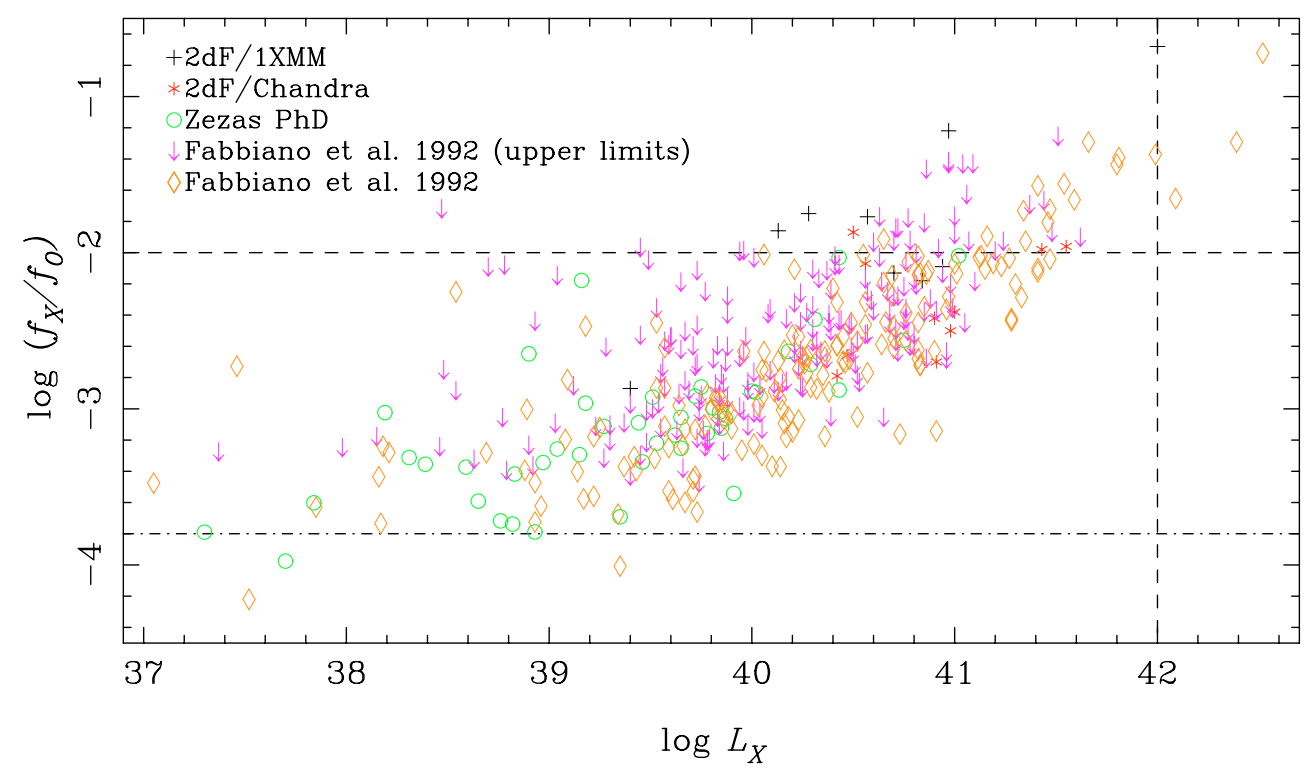

Fig. 1. Plot of $\log \left(f_{\mathrm{X}} / f_{\mathrm{O}}\right)$ versus $\log L_{\mathrm{X}}$. Plotted here are values calculated from our XMM-Newton and Chandra data, as well as from the literature (see legend in the plot). For all samples, only galaxies which are classified as H II or absorption-line are plotted. All luminosities are for the same energy band $(0.5-2.0 \mathrm{keV})$. The two dashed lines demarcate the regions of $\log \left(f_{\mathrm{X}} / f_{\mathrm{O}}\right)-L_{\mathrm{X}}$ space which may be inhabited mainly either by NGs $\left(\log L_{\mathrm{X}}<42, \log \left(f_{\mathrm{X}} / f_{\mathrm{O}}\right)<-2\right)$ or by AGN. The dash-dotted line indicates an estimate for $\log \left(f_{\mathrm{X}} / f_{\mathrm{O}}\right)$ from low mass X-ray binaries in a $10^{11} M_{\odot}$ galaxy (see text).

and $B-V=0.655$. The latter is the average $B-V$ value for nearby galaxies of all morphological types (Fukugita et al. 1995, Table 3a).

There is a clear correlation between the quantities plotted in the figure. From the relation $L_{\mathrm{X}} \sim L_{B}^{1.8}$ (Fabbiano et al. 1992), we would indeed expect a correlation of the form, roughly, $\log \left(f_{\mathrm{X}} / f_{\mathrm{O}}\right) \sim 0.5 \log L_{\mathrm{X}}$, as is the case in this plot.

The observed correlation shows that $\log \left(f_{\mathrm{X}} / f_{\mathrm{O}}\right)$ reaches values significantly higher than -2 as $L_{X}$ becomes higher than $\log L_{X} \approx 41$. Thus, any survey for NGs which uses a $\log \left(f_{\mathrm{X}} / f_{\mathrm{O}}\right)<-2$ cut is likely to suffer from incompleteness at the brightest luminosities. Although none of our samples can be said to be statistically complete, we may assume that galaxies from different populations are randomly selected. To assess the incompleteness, we give the fraction of NGs in three $L_{X}$ regions per NG type and sample in Table 3. Only NGs with $\log \left(f_{\mathrm{X}} / f_{\mathrm{O}}\right)>-2$ are included. Galaxies for which only upper limit values are available are not used.

From Fig. 1 and Table 3 it is clear that all galaxies in the sample of Zezas (2001) have both $\log L_{\mathrm{X}}<42$ and $\log \left(f_{\mathrm{X}} / f_{\mathrm{O}}\right)<-2$. However, in the sample of Fabbiano et al. (1992), there is a number of galaxies for which $\log \left(f_{\mathrm{X}} / f_{\mathrm{O}}\right)>-2$.

Specifically, as can be seen from Table 3 , there are 13 galaxies from this sample, for which $\log \left(f_{\mathrm{X}} / f_{\mathrm{O}}\right)>$ -2 and $\log L_{X}<42$. All but one of these galaxies have $41<\log L_{X}<42$. About $15 \%$ are starbursts $(2 / 13)$ and the rest massive ellipticals, brightest either in a group (BGGs) or cluster (BCGs). In the $\log L_{X} \geq 42$ region, there are 3 galaxies with $\log \left(f_{\mathrm{X}} / f_{\mathrm{O}}\right)>-2$. Two of these are massive elliptical BGGs and the third is a cD BCG.

It is instructive to investigate how many galaxies with $\log L_{\mathrm{X}}>42$ we should expect to detect, given our survey's flux limit and area covered. We used our flux limits and $\log L_{\mathrm{X}}>42$ to integrate the local X-ray luminosity function of NGs (Georgantopoulos et al. 2005). We found that we would expect to see $\lesssim 1$ galaxy with each telescope. In the $2 d F G R S$ correlation sample, there is a single absorption line galaxy at

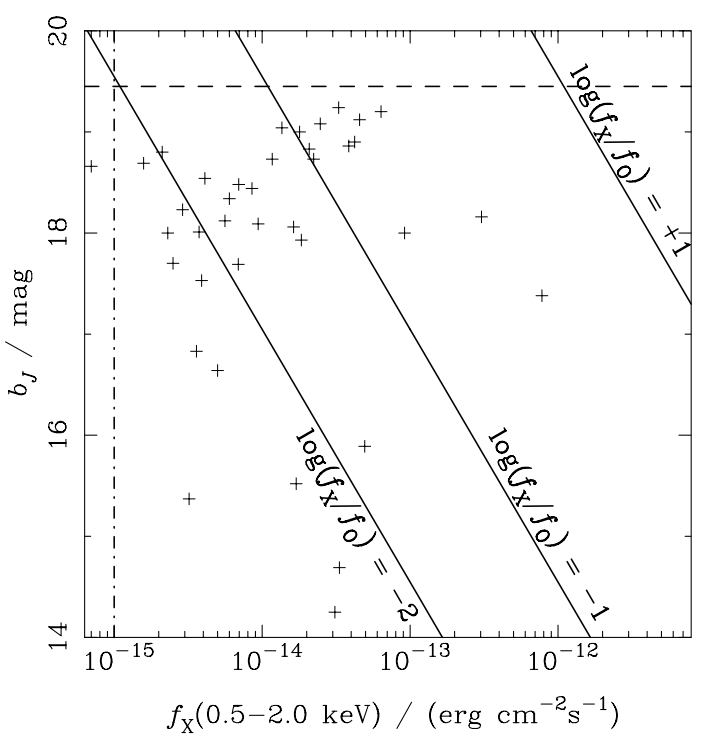

Fig. 2. Plot of $b_{\mathrm{J}}$ magnitude versus logarithmic X-ray flux for all galaxies in Tables 1 and 2 . The region to the right of the line $\log \left(f_{\mathrm{X}} / f_{\mathrm{O}}\right)=-1$ is expected to be occupied mainly by AGN. NGs are expected to be found mainly in the region to the left of the $\operatorname{line} \log \left(f_{\mathrm{X}} / f_{\mathrm{O}}\right)=-2$.

$\log L_{X}=42$, which is a good NG candidate. There is thus good order-of-magnitude agreement between our simple estimate and actual results.

It is also useful to compare observed values of $\log \left(f_{\mathrm{X}} / f_{\mathrm{O}}\right)$ to the numbers expected due to low mass X-ray binaries (LXRBs), whose combined luminosity has been shown to scale with the stellar mass of the host galaxy (Gilfanov 2004). We estimated the value of $\log \left(f_{\mathrm{X}} / f_{\mathrm{O}}\right)$ expected for a $10^{11} M_{\odot}$ galaxy, using the $L_{\mathrm{X}}(\mathrm{LXRB})-M_{*}$ and $M_{*} / L_{K}-(B-V)$ correlations from Gilfanov (2004) (Fig. 14 and Eq. (2), respectively). We used the average value of $B-V$ for all galaxies in our paper, and the average value of $V-K$ for all galaxy types from Mannucci et al. (2001). We obtained the value $\log \left(f_{\mathrm{X}} / f_{\mathrm{O}}\right)_{\mathrm{LXRB}}=-3.8$, shown by the 
dot-dashed line in Fig. 1. The bulk of the points in this plot fall above this line, suggesting that this value is a good estimate for a lower $\log \left(f_{\mathrm{X}} / f_{\mathrm{O}}\right)$ estimate.

Our sample is by no means complete. In Fig. 2, we plot $b_{\mathrm{j}}$ magnitude against $0.5-2.0 \mathrm{keV}$ flux for all galaxies in the $2 d F$ $G R S$ correlation sample. The solid oblique lines show $f_{\mathrm{X}}-b_{\mathrm{j}}$ loci for different values of $\log \left(f_{\mathrm{X}} / f_{\mathrm{O}}\right)$. Our galaxies are split in approximately equal numbers among the regions demarcated by the constant $\log \left(f_{\mathrm{X}} / f_{\mathrm{O}}\right)$ lines. The dashed horizontal line shows the $2 d F G R S$ magnitude limit at $b_{\mathrm{j}}=19.45$. The dashed-dotted vertical line shows the approximate flux limit of our correlation sample. It is clear that, at this limit, our survey misses galaxies in the region $-2<\log \left(f_{\mathrm{X}} / f_{\mathrm{O}}\right)<-1$, where luminous NGs are likely to be found. Such galaxies would not be missed at a higher flux limit, $\sim 2 \times 10^{-14} \mathrm{erg} \mathrm{cm}^{-2} \mathrm{~s}^{-1}$. Furthermore, for galaxies brighter than $b_{\mathrm{j}} \sim 16$, saturation effects affect the completeness of $2 d F G R S$ (Norberg et al. 2002).

The Fabbiano et al. (1992) sample also suffers from increasing incompleteness as $\log L_{\mathrm{X}}$ increases. Table 3 shows that the fraction of NGs with $\log \left(f_{\mathrm{X}} / f_{\mathrm{O}}\right)>-2$ rises from $\sim 8 \%$ (13 out of 167 galaxies) below $\log L_{X}=42$ to $100 \%$ above $\log L_{X}=42$ ( 3 out of 3 galaxies). For $\log L_{X}<42$, the majority of NGs with $\log \left(f_{\mathrm{X}} / f_{\mathrm{O}}\right)>-2$ are massive ellipticals, and for $\log L_{\mathrm{X}} \geq 42$, all are of this type. Although the $2 d F G R S$ correlation sample has fewer galaxies, it is clear that, at least at the highest luminosities, there is a similar pattern, with absorption line galaxies making up the bulk of NGs with $\log \left(f_{\mathrm{X}} / f_{\mathrm{O}}\right)>-2$. We note, however, that, considering all $L_{\mathrm{X}}$ regions together, the fraction of NGs with $\log \left(f_{\mathrm{X}} / f_{\mathrm{O}}\right)>-2$ in the $2 d F G R S$ correlation sample is much higher than in the Fabbiano et al. (1992) sample ( $\sim 40 \%$ versus $\sim 9 \%$ ). This may suggest that the $2 d F G R S$ correlation sample may suffer from residual AGN contamination. Furthermore, as mentioned in Sect. 3, this sample suffers from incompleteness at bright optical magnitudes.

Considering all samples, a picture is thus emerging in which the $\log \left(f_{\mathrm{X}} / f_{\mathrm{O}}\right)<-2$ criterion selects against the brightest ellipticals, but is not inadequate for other morphological types. However, the ellipticals in question, although "normal" in the sense that they do not host an AGN, belong to a distinct sub-class with respect to star-forming galaxies, as well as other ellipticals: these galaxies are found in the centres of X-ray bright groups or clusters. Such systems are affected significantly by their environment. For instance, they are, on average, considerably more luminous than normal ellipticals. Furthermore, group dominant galaxies have been shown to have temperature profiles indicative of central cooling (Helsdon \& Ponman 2000), leading to the suggestion that their halos are actually the product of cooling flows associated with the surrounding group.

The significance of the findings from the $2 d F G R S$ correlation samples is unclear, given the uncertainty in morphological type and diagnostic emission line ratios. Furthermore, classification of $2 d F G R S$ galaxies which remain unclassified in this work would make the current picture more clear. However, flux calibrated spectra of higher signal-to-noise ratios over the full wavelength range, covering all diagnostic emission lines, are necessary for this to be achieved.

From a different perspective, we note that we found no confirmed AGN with $\log \left(f_{\mathrm{X}} / f_{\mathrm{O}}\right)<-2$. Regardless of any completeness problems, this shows that the NHS surveys (Georgakakis et al. 2004b; Georgantopoulos et al. 2005) are at least not significantly contaminated by AGN.

\section{Summary}

We compiled a sample of galaxies observed with XMM-Newton and Chandra for which there are optical spectral observations in the $2 d F G R S$. We looked for X-ray luminous $\left(L_{\mathrm{X}}>10^{42} \mathrm{erg} \mathrm{s}^{-1}\right)$ NGs and assessed the empirical criterion $\log \left(f_{\mathrm{X}} / f_{\mathrm{O}}\right)<-2$ for separating NGs from AGN. Our main results are:

1. No luminous NGs were found.

2. Five H II galaxies and three absorption-line galaxies have $\log \left(f_{\mathrm{X}} / f_{\mathrm{O}}\right)>-2$.

We also carried out the same type of search in two samples from the literature. In the Zezas (2001) sample, all galaxies have $\log L_{\mathrm{X}}<42$ and $\log \left(f_{\mathrm{X}} / f_{\mathrm{O}}\right)<-2$. In the Fabbiano et al. (1992) sample, there are two starbursts and fourteen ellipticals with $\log \left(f_{\mathrm{X}} / f_{\mathrm{O}}\right)>-2$. This translates to an incompleteness of $\sim 8 \%$ (100\%) for $\log L_{X}<42\left(\log L_{X} \geq 42\right)$.

Considering all samples, we thus find that the $\log \left(f_{\mathrm{X}} / f_{\mathrm{O}}\right)<$ -2 criterion seems to select primarily against the brightest, massive ellipticals (BCGs and BGGs).

Further, we also find that the great majority of our galaxies have $\log \left(f_{\mathrm{X}} / f_{\mathrm{O}}\right)>-3.8$ which represents an estimate for the contribution of LXRBs to the X-ray luminosity of galaxies.

Acknowledgements. We thank the anonymous referee for constructive comments which helped improve the manuscript. This work is funded in part by the Greek National Secretariat for Research and Technology within the framework of the Greece-USA collaboration programme Study of Galaxies with the Chandra X-ray Satellite. We acknowledge the use of data from the $X M M-N e w t o n$ Science Archive at VILSPA, the Chandra-XAssist archive, and the 2dFGRS. This research has made use of data obtained from the High Energy Astrophysics Science Archive Research Center (HEASARC), provided by NASA's Goddard Space Flight Center. This research has made use of the NASA/IPAC Extragalactic Database (NED) which is operated by the Jet Propulsion Laboratory, California Institute of Technology, under contract with the National Aeronautics and Space Administration.

\section{References}

Alexander, D. M., Bauer, F. E., Brandt, W. N., et al. 2003, AJ, 126, 539 Baldwin, J. A., Phillips, M. M., \& Terlevich, R. 1981, PASP, 93, 5 Colless, M., Dalton, G., Maddox, S., et al. 2001, MNRAS, 328, 1039

Colless, M., Peterson, B. A., Jackson, C., et al. 2003

[arXiv: astro-ph/0306581]

Fabbiano, G. 1989, ARA\&A, 27, 87

Fabbiano, G., Kim, D.-W., \& Trinchieri, G. 1992, ApJS, 80, 531

Fukugita, M., Shimasaku, K., \& Ichikawa, T. 1995, PASP, 107, 945

Georgakakis, A., Georgantopoulos, I., Stewart, G. C., Shanks, T., \& Boyle, B. J. 2003, MNRAS, 344, 161

Georgakakis, A., Georgantopoulos, I., Vallbé, M., et al. 2004a, MNRAS, 349, 135

Georgakakis, A. E., Georgantopoulos, I., Basilakos, S., Plionis, M., \& Kolokotronis, V. 2004b, MNRAS, 354, 123

Georgantopoulos, I., Georgakakis, A., \& Koulouridis, E. 2005, MNRAS, 360, 782

Giacconi, R., Zirm, A., Wang, J., et al. 2002, ApJS, 139, 369

Gilfanov, M. 2004, MNRAS, 349, 146

Gilfanov, M., Grimm, H.-J., \& Sunyaev, R. 2004, MNRAS, 347, L57

Helsdon, S. F., \& Ponman, T. J. 2000, MNRAS, 315, 356

Ho, L. C., Filippenko, A. V., \& Sargent, W. L. W. 1997, ApJS, 112, 315

Hornschemeier, A. E., Bauer, F. E., Alexander, D. M., et al. 2003, AJ, 126, 575

Levenson, N. A., Weaver, K. A., \& Heckman, T. M. 2001, ApJ, 550, 230

Mannucci, F., Basile, F., Poggianti, B. M., et al. 2001, MNRAS, 326, 745

Moran, E. C., Lehnert, M. D., \& Helfand, D. J. 1999, ApJ, 526, 649

Norberg, P., Cole, S., Baugh, C. M., et al. 2002, MNRAS, 336, 907

Norman, C., Ptak, A., Hornschemeier, A., et al. 2004, ApJ, 607, 721

Ptak, A., \& Griffiths, R. 2003, in Astronomical Data Analysis Software and Systems XII, ASP Conf. Ser., 295, 465

Stevens, I. R., Read, A. M., \& Bravo-Guerrero, J. 2003, MNRAS, 343, L47

Stocke, J. T., Morris, S. L., Gioia, I. M., et al. 1991, ApJS, 76, 813

Veilleux, S., \& Osterbrock, D. E. 1987, ApJS, 63, 295

Zezas, A. L. 2001, Ph.D. Thesis, Univ. of Leicester

Zezas, A. L., Georgantopoulos, I., \& Ward, M. J. 1998, MNRAS, 301, 915 